\title{
pH Dependence of the Dissolution Rate of Enteric- Coated Drug Spheres Determined by Broadband Acoustic Resonance Dissolution Spectroscopy (BARDS)
}

\author{
Rachel Evans-Hurson ${ }^{1}$, Seán McSweeney ${ }^{1}$, Bastiaan Vos ${ }^{1}$, Jacob Krüse ${ }^{2}$, \\ J. J. Keating ${ }^{1,3}$, and Dara Fitzpatrick ${ }^{1, *}$ \\ ${ }^{1}$ Department of Chemistry, Analytical and Biological Chemistry Research Facility (ABCRF), University College Cork, Ireland \\ ${ }^{2}$ Kinetox, Beilen, The Netherlands \\ ${ }^{3}$ School of Pharmacy, University College Cork, Ireland
}

\begin{abstract}
Enteric coatings are widely used in formulations of drug delivery spheres. The coating protects an active pharmaceutical ingredient (API) from acidic conditions in the low pH environment of the stomach. The coating breaks down readily at higher $\mathrm{pH}$ in the lower intestine to allow absorption of the API. The thickness of the enteric coating is one of the factors that determine the release rate of the drug in the gastrointestinal tract. It is difficult to determine the loading of the drug layer and enteric coating on the core support sphere without conventional dissolution testing during and post manufacture. Broadband acoustic resonance dissolution spectroscopy (BARDS) potentially offers a new, rapid approach to characterizing enteric coatings during their manufacture. BARDS applications are based on reproducible changes in the compressibility of a solvent during dissolution, which is monitored acoustically via associated changes in the frequency of induced acoustic resonances. Two drug sphere formulations that yield characteristic and reproducible data were investigated. A steady-state acoustic lag time is associated with the disintegration of the enteric coating and drug layer in basic solution. This lag time is $\mathrm{pH}$ dependent and is indicative of the rate at which the coating and layers dissolve. BARDS analysis has the potential to characterize drug sphere formulations at-line in very short timescales. BARDS represents a complementary technique to conventional dissolution testing that could be used in precompliance testing for quality assurance during manufacture. BARDS data, in the future, may also be indicative of the likely performance of a formulation under USP dissolution testing.
\end{abstract}

KEYWORDS: Hypromellose; enteric-coated spheres; dissolution; BARDS.

\section{INTRODUCTION}

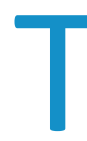

he trend toward the use of drug- and enteric-coated sugar spheres for controlled oral delivery is driven by increased efficacy and ease of formulation of different dosage levels. However, difficulties exist in determining the thickness of drug layer and enteric coatings in a time-efficient manner during manufacture and post production. The thickness of the drug layer determines dosage of the API, and the thickness and grade of the enteric coating determines the timing of release of the drug in the gastrointestinal tract. There are three commonly used types of enteric coatings, namely, polymethacrylates, cellulose esters, and polyvinyl derivatives. In a previous study (1), a prescribed drug product Lanzol that contains enteric-coated drug delivery spheres was investigated. The API lansoprazole is coated on a core sugar sphere that is then coated with a methacrylic acid-ethyl acrylate copolymer (Eudragit) before encapsulation. The study demonstrated unique BARDS profiles for individual size distributions of (noncoated) sugar spheres. The coated spheres displayed a lag time associated with the thickness of the drug and enteric coatings. The lag time showed a dependence on the concentration of sodium hydroxide in the dissolution medium. An ability to determine the ratio of placebo versus coated spheres was also demonstrated.

In the current study, a comparison was firstly made between Lanzol (Rowa Pharma) and another drug product Cymbalta (Eli Lilly), which is coated with a different enteric material called hypromellose acetate succinate (HPMCAS). This has been used as an enteric coating since 1986. The API and excipients of both formulations are shown in Table 1. This was followed by an investigation of solution gas oversaturation brought about by the dissolution of the Cymbalta formulation. BARDS analysis is a new approach used to monitor the intrinsic acoustic signature profiles of the core spheres and the coated product as they dissolve. The BARDS signal results from reproducible changes in the compressibility of a solvent

*Corresponding author. 
during dissolution. The changes in compressibility alter the speed of inducted sound in the vessel, resulting in frequency changes within the solution.

Table 1. Formulation Details of Cymbalta and Lanzol Obtained from the Packaging Information Leaflet

\begin{tabular}{|c|l|l|}
\hline & Lanzol & Cymbalta \\
\hline API & Lansoprazole & Duloxetine (as HCl) \\
\hline \multirow{5}{*}{ Excipients } & Eudragit L 30-D 55 & $\begin{array}{l}\text { Hypromellose acetate } \\
\text { succinate (HPMCAS) }\end{array}$ \\
\cline { 2 - 3 } & Hypromellose & Hypromellose \\
\cline { 2 - 3 } & Meglumine & Sucrose \\
\cline { 2 - 3 } & $\begin{array}{l}\text { Sugar spheres (sucrose } \\
\text { and maize starch) }\end{array}$ & $\begin{array}{l}\text { Sugar spheres (sucrose } \\
\text { and maize starch) }\end{array}$ \\
\cline { 2 - 3 } & Mannitol (E421) & \\
\cline { 2 - 3 } & Titanium dioxide (E171) & Titanium dioxide (E171) \\
\cline { 2 - 3 } & Sodium lauryl sulfate & Triethyl citrate \\
\cline { 2 - 3 } & Macrogol 6000 & Talc \\
\cline { 2 - 3 } & Polysorbate 80 & \\
\hline
\end{tabular}

The principles underlying the BARDS response are as follows. The sound velocity $(v)$ in a medium, whether air or liquid phase, is determined by eq 1 :

$$
v_{\text {(sound) }}=\sqrt{\frac{1}{K \cdot \rho}}
$$

where $\rho$ is mass density and $K$ is compressibility, which is the inverse of the bulk modulus of the medium. Generation of micro gas bubbles in a liquid decreases the density in a negligible way in comparison with the large increase in compressibility. The net effect is a significant reduction of the sound velocity in the liquid. The following relationship between the fractional bubble volume and the sound velocity in water was derived by Crawford (2) as given in eq 2:

$$
\frac{v_{w}}{v}=\sqrt{\left(1+1.49 \times 10^{4} \cdot f_{a}\right)}
$$

where $v_{w}$ and $v$ are the sound velocities in pure and bubble-filled water, respectively, and $f_{a}$ is the fractional volume occupied by air bubbles. The factor $1.49 \times 10^{4}$ in eq 2 was calculated as shown in eq 3 :

$$
\left(v_{w}\right)^{2} \rho_{w} \cdot \frac{1}{y p}=1.49 \times 10^{4}
$$

where $\rho_{w}$ is the density of water, $\gamma$ is the ratio of specific heats for dry air, and $p$ is the atmospheric air pressure. Equation 2 is based on the approximation presented originally by Wood (3).

BARDS analysis of the induced acoustic resonances of the dissolution vessel is focused on the lowest variable frequency time course (i.e., the fundamental resonance mode of the liquid). The vessel is induced to resonate through simple mechanical striking of the magnetic stir bar on the internal wall.

The fundamental resonant frequency is determined by the sound velocity in the liquid and the approximate but fixed height of the liquid level, which corresponds to one quarter of its wavelength. The frequency response is described as:

$$
f r e q=\frac{f r e q_{w}}{\sqrt{1+1.49 \times 10^{4} \cdot f_{a}}}
$$

where fre $q_{w}$ and freq are the resonance frequencies of the fundamental resonance modes in pure and bubble-filled water, respectively. The transient total volume of the gas bubbles is determined by introduced entrained gas bubbles, bubbles evolving due to gas oversaturation, and bubbles disappearing due to elimination at the surface or reabsorption of the gas. A detailed and comprehensive outline of the principles and underlying processes involved in BARDS analysis is given by Fitzpatrick et al. (4). The reproducibility of the acoustic phenomenon used in BARDS has been also demonstrated in a recent study by Trávníček et al. (5). Several other references (6-11) to the phenomenon have appeared intermittently in the literature over the decades.

Figure 1 shows a BARDS spectrum of the dissolution in 25 $\mathrm{mL} 0.01 \mathrm{M} \mathrm{NaOH}$ of $0.15 \mathrm{~g}$ of Cymbalta drug spheres that have been removed from their capsules. The acoustic profile of interest is called the fundamental curve. The frequency minimum $\left(f_{\min }\right)$ represents an equilibrium between the rate of formation of gas in solution and the rate of gas liberation at the surface. In BARDS analysis, the fundamental curve is used to make comparisons between individual experiments. Note that the acoustic frequencies of the vessel remain steady for the first

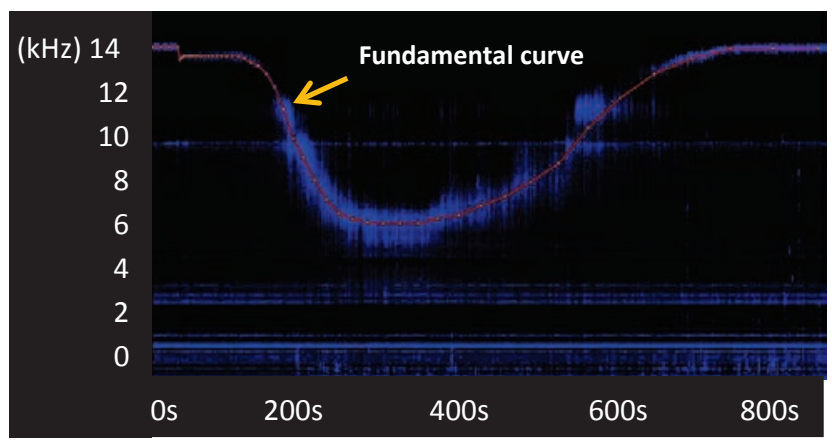

Figure 1. BARDS spectrogram of the dissolution of $0.15 \mathrm{~g}$ of Cymbalta in $25 \mathrm{~mL} 0.01 \mathrm{M} \mathrm{NaOH}$ solution. Note the spheres are added at 30-s time point. 
$30 \mathrm{~s}$ until the addition of the spheres. Thereafter, the resonant frequency at $14.5 \mathrm{kHz}$ decreases to $6 \mathrm{kHz}$ and gradually returns to steady state after $\approx 700 \mathrm{~s}$. The constant frequency at $10 \mathrm{kHz}$ is one of many resonant frequencies of the vessel that is not dependent on the liquid compressibility and therefore remains unchanged.

In general, gases entrained between and within particles are introduced into a solution when a compound is dissolved in an aqueous solvent. Also, a reduction in the solubility of gases in solution will take place, resulting in gas oversaturation. This oversaturation is partly removed by generation of gas bubbles in the solution. The entrainment and liberation of gas bubbles and their subsequent escape from the solution causes a transient yet reproducible change in the compressibility of the solution that can be monitored acoustically under standardized conditions.

\section{EXPERIMENTAL}

\section{Materials}

Analar grade sodium hydroxide was purchased from Sigma Aldrich. Lanzol product (Lot \# LC 10100), manufactured by Rowa Pharmaceuticals (Bantry, County Cork, Ireland), was obtained from a local pharmacy. Cymbalta (Lot \# C189795), manufactured by Eli Lilly, was also obtained from a local pharmacy. Doubly distilled water $\left(\mathrm{dH}_{2} \mathrm{O}\right)$ with a resistivity of $18 \mathrm{M} \Omega \mathrm{cm}^{-1}$ was used for the preparation of all solutions used in experiments. Suglets $(600-710-\mu \mathrm{m}$ diameter sugar spheres) were obtained from Colorcon.

\section{Instrumentation}

A custom prototype spectrometer was built and used to investigate the BARDS responses $(12,13)$. It consists of a chamber with a glass dissolution vessel, a microphone (Sony ECM-CS10, range 100-16 kHz), a magnetic stirrer, and a stir bar. There is access at the front to the dissolution vessel and at the top to place a sample in a weighing boat on a tipper motor for introduction of the formulation. The microphone is positioned above the top of the glass within the housing. The glass containing $25 \mathrm{~mL}$ of solvent is placed on the stirrer plate. The stirrer motor underneath is positioned so as to allow the magnetic stir bar to gently tap the inner glass wall. In this way, the stir bar acts as a source of broadband acoustic excitation, thereby inducing various acoustic resonances in the glass, the liquid, and the air column above the liquid. The audio is sampled at a rate of $44.1 \mathrm{kHz}$. A fast Fourier transform is applied to the signal. The resonances of the liquid vessel are recorded in a frequency band of $0-20 \mathrm{kHz}$. A frequency time course is generated as shown in Figure 1. The spectrometer has no similarity to a typical USP setup.

\section{Experimental Procedure}

The spectrometer records the steady state resonances of the system as a reference for thirty seconds when the stirrer is set in motion. The Cymbalta spheres were removed from the capsule prior to introducing them to the solution. The pitch of the resonance modes in the solution changed significantly when the spheres were added before gradually returning to steady state over several minutes. The amount of spheres added is expressed by mass in all figures. Solutions were gas equilibrated prior to use through agitation by shaking vigorously for $60 \mathrm{~s}$ and then resting for $10 \mathrm{~min}$.

The frequency time course of the fundamental resonance is presented as manually extracted data from the total acoustic response. Spectra were recorded for 300-800 sec. All experiments were performed in duplicate, and average readings with error bars representing the data spread are presented. The time courses of the observed acoustic profiles were highly reproducible under standardized conditions of constant volume, mass, temperature, and stirring rate (12). In general, the dissolution process is complete at the frequency minimum. Variability can increase post frequency minimum with erratic outgassing in the presence of particulates in suspension, which impede egress of gas to the surface. Variability may also be a consequence of clotting at the bottom of the vessel, thereby resulting in an undefined viscous layer that may also hamper proper stirring. Such a diffuse layer may prevent sharp resonances because of the lack of a well-defined reflection wall at the bottom.

The steady-state frequency before addition of the formulation is designated as the "volume line," so called as it varies depending on the liquid volume in the vessel.

\section{RESULTS AND DISCUSSION}

Figure 2A shows the BARDS spectra of the drug products Cymbalta and Lanzol. Each curve represents two separate experiments. The drug spheres were removed from the capsules for analysis and added to aqueous solution after $30 \mathrm{sec}$ of stirring. The Cymbalta curve immediately dropped approximately $300 \mathrm{~Hz}$ to a plateau frequency of 
$14 \mathrm{KHz}$. This indicates a slight increase in the compressibility of the solution due to the presence of the drug spheres. Gas trapped in the spheres contributed to compressibility change but was unable to escape. This frequency was sustained for about $100 \mathrm{~s}$ as the enteric and drug layers dissolve. This lag time is indicative of the rate at which the coating and drug layer dissolved. Thereafter, the microporous core spheres were exposed and dissolved, releasing previously incompressible trapped gas into the solution. This increased the compressibility further, resulting in the significant decrease of the fundamental frequency. The frequency reached a minimum at $\approx 250$ s. At this point, the rate of gas liberation/evolution became equal to the rate of loss of gas at the surface. The frequency began to return to steady state at $380 \mathrm{~s}$ and was reached at $\approx 650 \mathrm{~s}$. Fitzpatrick et al. (1) demonstrated how uncoated spheres dissolve instantaneously upon addition to the solvent. It is not until the layers and coating are added that a lag time becomes apparent. Figure 3 shows a typical Cymbalta sphere measuring $\approx 1 \mathrm{~mm}$ in diameter.
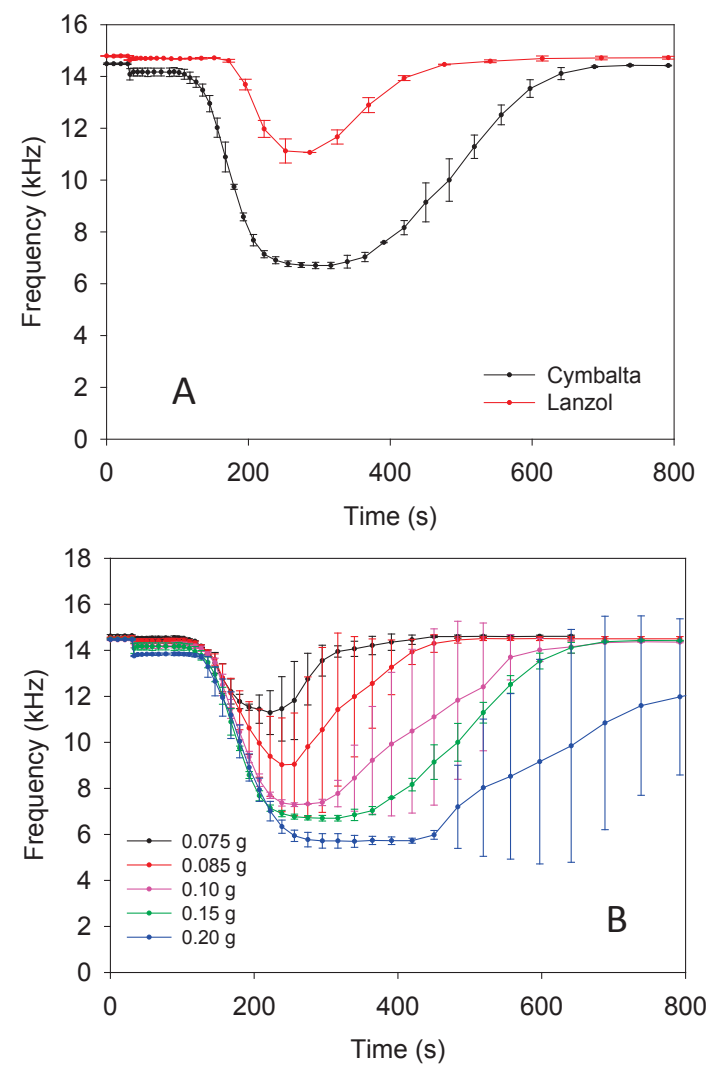

Figure 2. (A) BARDS profiles of the dissolution of $0.15 \mathrm{~g}$ Cymbalta and Lanzol in $0.01 \mathrm{M} \mathrm{NaOH}$ and (B) concentration profiles of Cymbalta samples with increasing mass dissolved in $0.01 \mathrm{M} \mathrm{NaOH}$.
The enteric coating used on Cymbalta spheres is HPMCAS, which is a hydroxypropyl methylcellulose with both acetyl and succinoyl substituted ester groups. The carboxylic acid groups on the succinoyl groups are ionized above a $\mathrm{pH}$ of 5.5-6.8, allowing the dissolution of the drug sphere to occur in the duodenum. Therefore, increasing the $\mathrm{pH}$ also increases the solubility of the enteric coating.

The lag time has the potential to be used during the layering process to indicate the thickness of the drug layer and enteric coating. Only finished product was available for this study; however, spray-dried formulations currently being prepared will be reported in a future study.

Lanzol demonstrated a profile to similar that of Cymbalta; however, the lag time for the dissolution of the coating and drug layer continued to $200 \mathrm{~s}$ in Figure 2A. The core spheres, when exposed to the solution, showed a reduced evolution of gas in comparison with the core spheres of Cymbalta. The return to steady state was reached $\approx 200 \mathrm{~s}$ earlier than for Cymbalta. Therefore, the two drug products each produced a unique acoustic profile related to their formulation and the types of core sphere incorporated in the formulation.

Figure $2 \mathrm{~B}$ shows a concentration profile of Cymbalta. Post addition of the spheres (30 s), the resonant frequency dropped slightly and incrementally with increasing mass of sample. The lag time remained constant due to the base

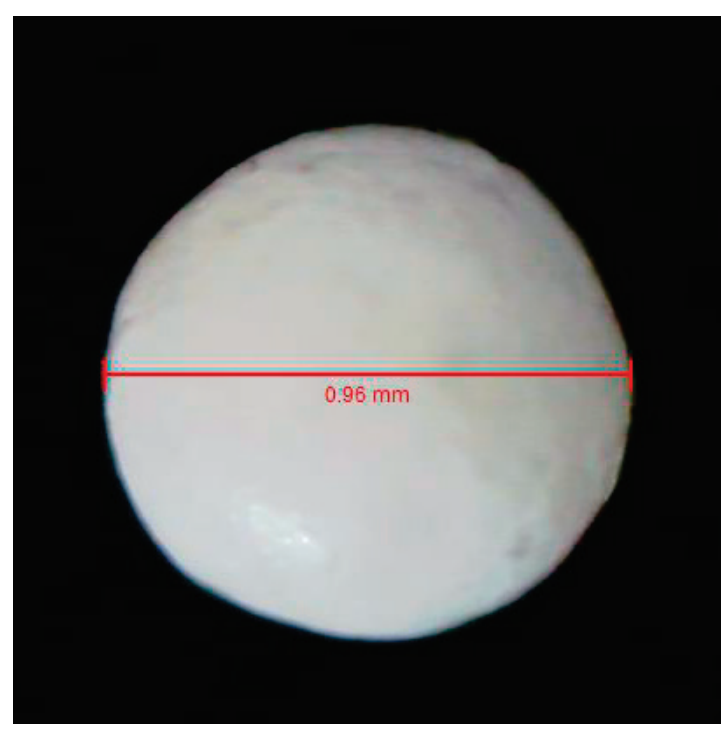

Figure 3. Single Cymbalta sphere measuring $\approx 1 \mathrm{~mm}$ diameter. 
concentration remaining the same for each experiment. This indicates that the rate at which the layers dissolved is independent of the sample mass; this is assuming the hydroxide concentration is always constant. The frequency plateau from 250 to $450 \mathrm{~s}$ for the blue profile $(0.20 \mathrm{~g})$ implies that the rate of gas evolution is equal to the rate of gas loss from the surface of the solution. The defined $f_{\min }$ at lower sample mass was gradually replaced with a frequency plateau with increasing sample mass. This also indicates the spheres persisted longer at higher sample mass leading to prolonged availability of nucleation sites for outgassing. The standard error increased post $f_{\min }$ for all sample masses except the green profile $(0.15 \mathrm{~g})$. This may be due to the final disintegration of the core spheres differing slightly between samples as mentioned previously.

Figure 4 shows the acoustic profiles of $0.15 \mathrm{~g}$ Cymbalta in $25 \mathrm{~mL}$ citric acid-disodium hydrogen phosphate buffer at varying $\mathrm{pH}$. The enteric coating failed to break down at low $\mathrm{pH}$ values of 3 and 4.8. The data indicate a very gradual breakdown of layers at $\mathrm{pH} 5$, which is illustrated by a response at $500 \mathrm{~s}$ when the core sphere was gradually exposed. The coating broke down more rapidly as the $\mathrm{pH}$ increased further. Figure 5 illustrates a significant correlation between the lag time recorded and the hydrogen ion concentration. Figure 6 shows the acoustic profiles of the aqueous dissolution of Cymbalta with increasing hydroxide concentration. The $\mathrm{pH}$ of water was high enough for disintegration of the layers to take place. The rate of disintegration of the layers increased with increasing $\mathrm{pH}$ of the solvent. The spectra in water are also better quality than those obtained in buffer; however, reproducibility is poor for some hydroxide concentrations tested.

Figure 7 shows fractional gas volume profiles calculated from the data shown in Figure 6 using eq 4. Crawford (2) and Woods (3) derived the relationship between the resonant frequency and the gas volume evolved during dissolution. The peak gas volume for the dissolution of $0.25 \mathrm{~g}$ of Cymbalta was roughly the same in water and in low concentrations of $\mathrm{NaOH}$. However, the peak gas volume was attained in a shorter time frame with increasing concentration of base. The absolute gas volume $(\mathrm{mL})$ can be acquired by multiplying the data in Figure 7 by the dissolution volume.

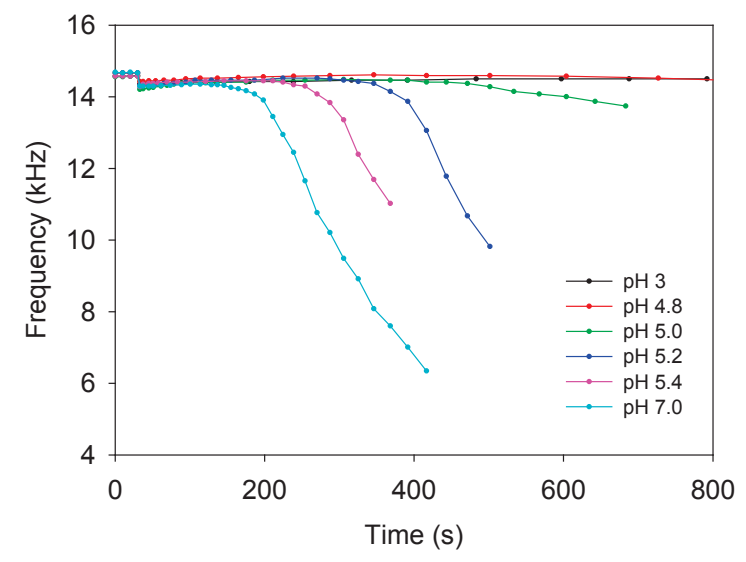

Figure 4. Acoustic profiles of $0.15 \mathrm{~g}$ Cymbalta in $25 \mathrm{~mL}$ citric aciddisodium hydrogen phosphate buffer at varying $\mathrm{pH}$.

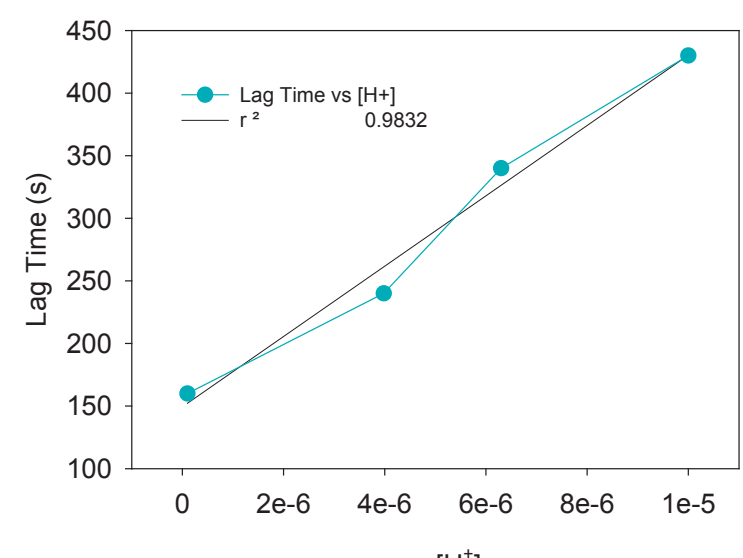

$\left[\mathrm{H}^{+}\right]$

Figure 5. Graph of lag time versus $\mathrm{H}+$ concentration as derived from data in Figure 4.

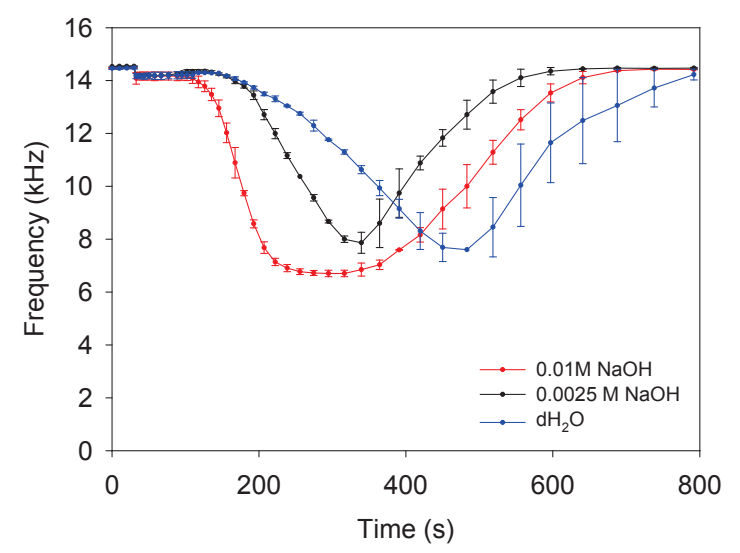

Figure 6. Comparison of the dissolution of $0.15 \mathrm{~g}$ Cymbalta in varying concentrations of $\mathrm{NaOH}$. 


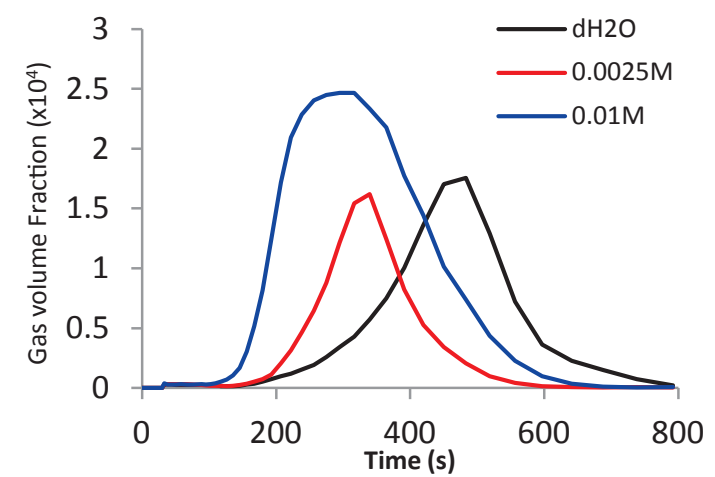

Figure 7. Comparison of fractional gas volume fractions of $0.15 \mathrm{~g}$ Cymbalta in varying concentrations of $\mathrm{NaOH}$.

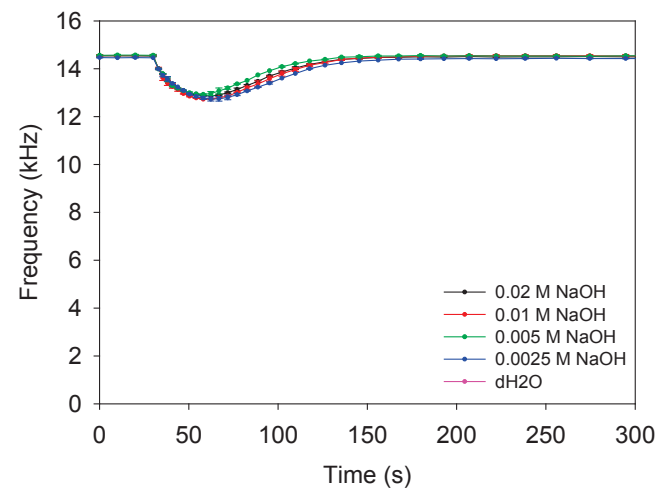

Figure 8. Acoustic profiles of $0.15 \mathrm{~g} 600-710-\mu \mathrm{m}$ sugar spheres in varying concentrations of $25 \mathrm{~mL} \mathrm{NaOH}$.

The data in Figure 8 show there is no significant effect on the response of uncoated sugar spheres upon varying the base concentration. The spheres are estimated to be of similar size as those used in the Cymbalta formulation. The source of the sugar spheres used in the formulation is unknown; therefore, surrogate spheres were used for demonstration purposes.

Figure 9A compares the response of the Cymbalta spheres and sugar spheres to a 50:50 mixture of both at $0.15 \mathrm{~g}$. The mixture demonstrates a bimodal response; the initial response is due to the placebo sugar spheres, and the delayed response is due to the Cymbalta-coated spheres. The initial response of the 50:50 mixture was not intermediate between the Cymbalta and sugar sphere responses; however, the second response was intermediate. Analysis of the contribution of the individual components of the 50:50 mixture indicates that the response of the placebo sugar spheres was "boosted" (Figure 9B). The response of the Cymbalta spheres in the
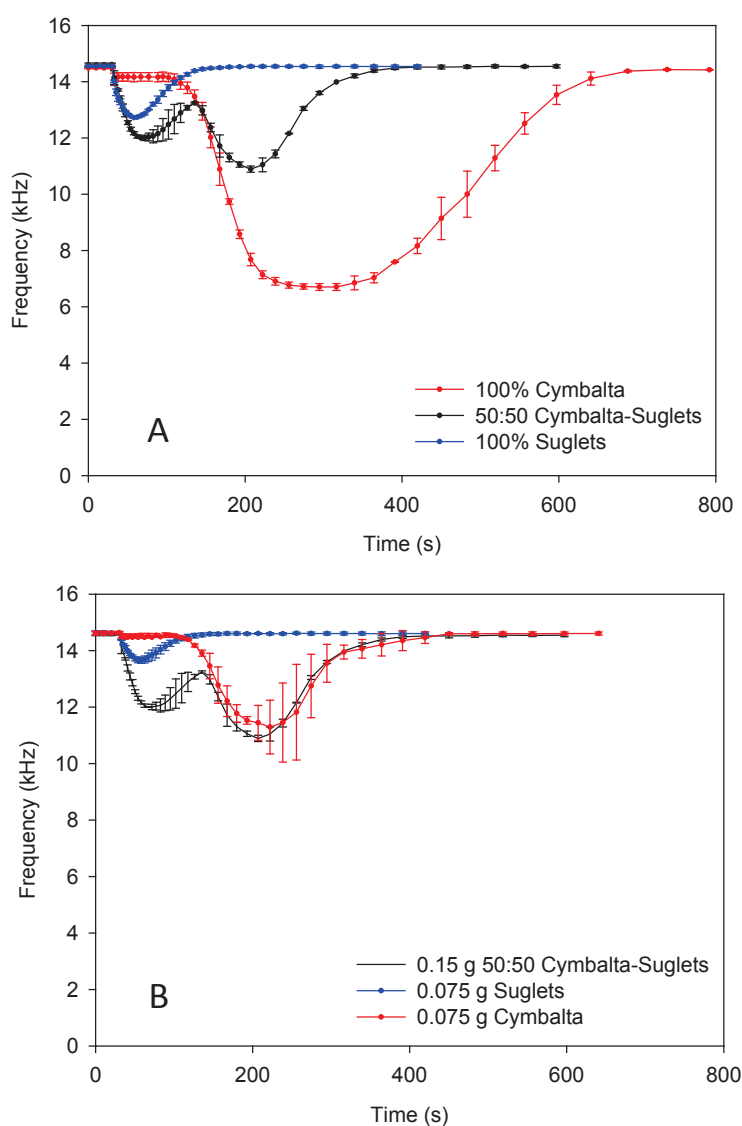

Figure 9. BARDS spectra of the dissolution of (A) $0.15 \mathrm{~g}$ Cymbalta, $0.15 \mathrm{~g}$ of 600-710 sugar spheres, and a 50:50 mixture of both spheres and Cymbalta with a mass of $0.15 \mathrm{~g}$, and (B) comparison of $0.15 \mathrm{~g}$ of 50:50 mixture of Cymbalta-sugar spheres with the individual components in $0.01 \mathrm{M} \mathrm{NaOH}$.

mixture remained largely unaffected by the presence of the uncoated sugar spheres. The increased response for the sugar spheres is most likely due to gas oversaturation generated during the disintegration of the layers on the coated Cymbalta spheres.

Gas oversaturation is indeed demonstrated by the dissolved oxygen (DO) measurement shown in Figure 10. The apparent DO levels reached a peak, which coincides with the end of the lag time, after which the level reduced as the solution began to outgas via bubble nucleation and growth. The rate of decrease slowed just after the $f_{\min }$ of the BARDS response after $300 \mathrm{~s}$. The DO level reached steady state at the same time point as the BARDS response also reached steady-state frequency. Note that the DO measurements were performed under nonequilibrium conditions, which means in this case, that the gas solubility was reduced during the lag time phase due to dissolution of the layers. However, since the amount of dissolved gas (oxygen) was not instantly equilibrating with 


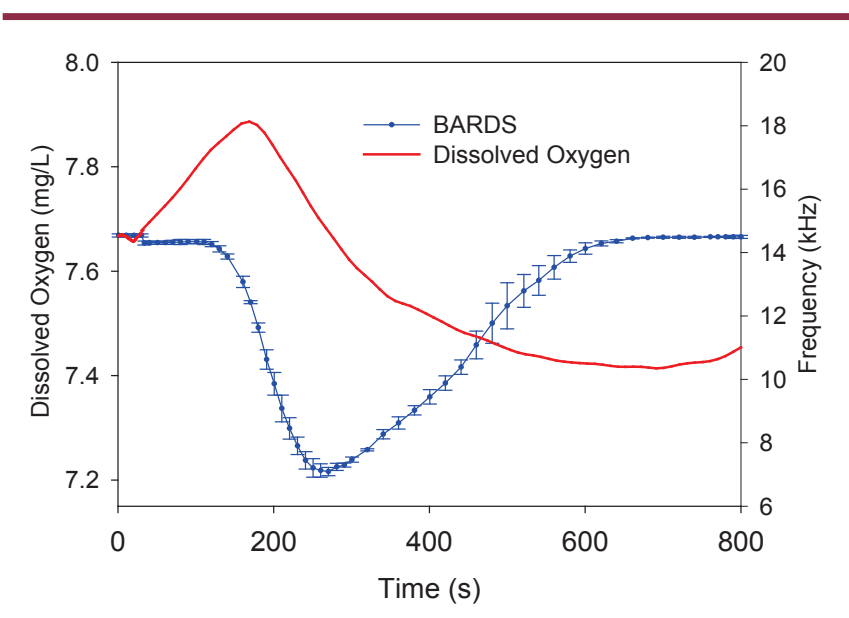

Figure 10. Comparison graph of dissolved oxygen profile and BARDS profile of $0.15 \mathrm{~g}$ Cymbalta spheres in $0.01 \mathrm{M} \mathrm{NaOH}$.

the atmosphere above the solution, a gas oversaturation and therefore a gas overpressure was created in the solution. Since the DO measurement is based on oxygen gas pressure in the solution, the induced overpressure is reflected in the measurement of an apparent increase in the DO concentration level. The level of BARDS overresponse for the placebo sugar spheres therefore depends on the amount of oversaturation induced by the disintegration of the layers and the number of available nucleation sites provided during the dissolution of the sugar sphere.

Figure 11 shows the BARDS profile associated with the addition of sugar spheres followed by the sequential addition of Cymbalta. The profile for the sequential addition in reverse order is also shown for comparison. The response of both the sugar spheres and the drug spheres remained the same regardless of the sequence of addition. The spheres and drug spheres were added at the same time point at either $30 \mathrm{~s}$ or $800 \mathrm{~s}$. The latter time point allows for the spectra to return to steady state. The spectra indicate that neither the spheres nor the drug-coated spheres allowed gas oversaturation post dissolution. Therefore, the over-response of the sugar spheres was due to a mutual interaction between the components of the mixture (i.e., the drug spheres provided gas oversaturation and the sugar spheresd provide gas nucleation sites). This interaction can only occur when both components are added simultaneously and not sequentially.

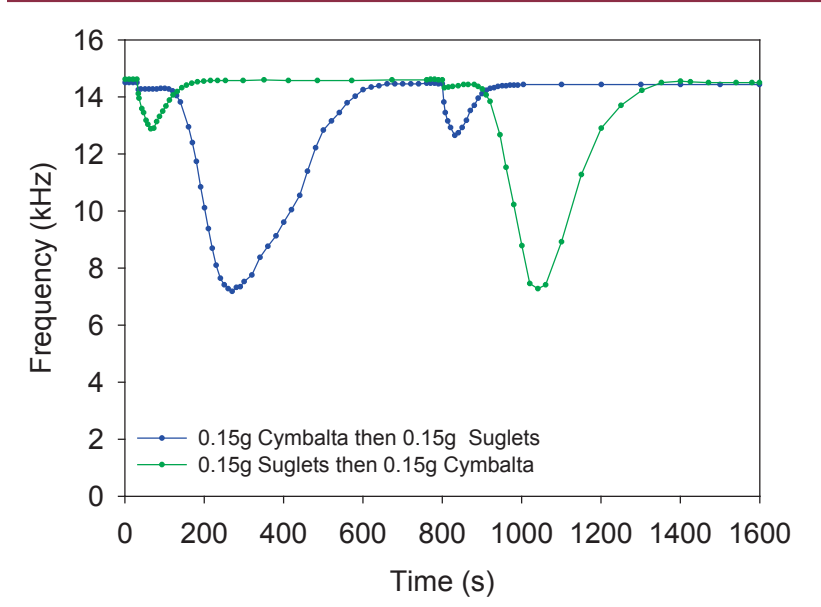

Figure 11. BARDS profiles of sequential addition of $0.15 \mathrm{~g}$ sugar spheres followed by $0.15 \mathrm{~g}$ Cymbalta and reverse order in $0.01 \mathrm{M} \mathrm{NAOH}$.

\section{CONCLUSIONS}

The data present a new way of investigating drug delivery sphere formulations during and post production in a rapid manner. Products with different formulations have a characteristic and intrinsic signature profile. The breakdown of the enteric coating is $\mathrm{pH}$ dependent in accordance with the $\mathrm{p} K_{a}$ of the polymer. Dissolved oxygen measurements have proved that an induced gas oversaturation is associated with the disintegration of the layers on the spheres. The oversaturation is relieved due to gas-bubble nucleation when the core sphere dissolves. BARDS data is a good indicative method to follow the physical decomposition of the drug sphere formulations and may therefore become a good indicator of how likely the product will behave under standard dissolution testing.

\section{CONFLICT OF INTEREST}

No conflict of interest has been declared by the authors.

\section{ACKNOWLEDGMENTS}

We wish to thank Enterprise Ireland and the ERDF for funding this research under contracts TD-2009-0327 and TD-2011-1069. We also thank the Irish Research Council for Ph.D. funding for Rachel Evans-Hurson and Colorcon for samples of sugar spheres (suglets).

\section{REFERENCES}

1. Fitzpatrick, D.; Evans-Hurson, R.; Fu, Y.; Burke, T.; Krüse, J.; Vos, B.; McSweeney, S. G.; Casaubieilh, P.; and Keating, J. J. Rapid profiling of enteric coated drug delivery spheres via Broadband Acoustic Resonance 
Dissolution Spectroscopy (BARDS). Analyst 2014, 139 (5), 1000-1006. DOI: 10.1039/C3AN01809A.

2. Crawford, F. S. The hot chocolate effect. Am. J. Phys. 1982, 50 (5), 398-404. DOI: 10.1119/1.13080.

3. Wood, A. B. A Textbook of Sound: Being an account of the Physics of Vibrations with special reference to recent theoretical and technical developments; MacMillan: New York, 1930.

4. Fitzpatrick, D.; Krüse, J.; Vos, B.; Foley, O.; Gleeson, D.; O'Gorman, E.; O'Keefe, R. Principles and Applications of Broadband Acoustic Resonance Spectroscopy (BARDS): A Sound Approach for the Analysis of Compounds. Anal. Chem. 2012, 84 (5), 2202-2210. DOI: 10.1021/ac202509s.

5. Trávníček, Z.; Fedorchenko, A. I.; Pavelka, M.; Hrubý, J. Visualization of the hot chocolate sound effect by spectrograms. J. Sound Vib. 2012, 331 (25), 53875392. DOI:10.1016/j.jsv.2012.07.038.

6. Farrell, W. E; McKenzie, D. P.; Parker, R. L. On the note emitted from a mug while mixing instant coffee. Math. Proc. Cambridge Philos. Soc. 1969, 65 (1), 365367. DOI: $10.1017 / \mathrm{S} 0305004100044303$.

7. Walker, J. The Amateur Scientist: Wonders of physics that can be found in a cup of coffee or tea. Sci. Am. 1977, 237 (5), 152-160. DOI: 10.1038/ scientificamerican1177-152.

8. Bragg, W. The World of Sound; Dover Publications,
Inc.: New York, 1968; p 158.

9. Drenckhan, W. Generation of Superstable, Monodisperse Microbubbles using a pH-Driven Assembly of Surface-Active Particles. Angew. Chem., Int. Ed. 2009, 48 (29), 5245-5247. DOI: 10.1002/ anie.200901531.

10. Devaud, M.; Hocquet, T.; Bacri, J.-C.; Leroy, V. The Minnaert bubble: an acoustic approach. Eur. J. Phys. 2008, 29 (6), 1263-1285. DOI: 10.1088/01430807/29/6/014.

11. Wilson, P. S.; Roy, R. A. An audible demonstration of the speed of sound in bubbly liquids. Am. J. Phys. 2008, 76 (10), 975-981. DOI: 10.1119/1.2907773.

12. Fitzpatrick, D.; Evans-Hurson, R.; Krüse, J.; Vos, B.; McSweeney, S.; Casaubieilh, P.; O'Gorman, E. The relationship between dissolution, gas oversaturation and outgassing of solutions determined by Broadband Acoustic Resonance Dissolution Spectroscopy (BARDS). Analyst 2013, 138, 5005-5010. DOI: 10.1039/C3AN36838F.

13. Fitzpatrick, D.; Scanlon, E.; Krüse, J.; Vos, B.; EvansHurson, R.; Fitzpatrick, E.; McSweeney, S. Blend uniformity analysis of pharmaceutical products by Broadband Acoustic Resonance Dissolution Spectroscopy (BARDS). Int. J. Pharm. 2012, 438 (1-2), 134-139. DOI: 10.1016/J.ljpharm.2012.07.073. 